\title{
Linx
}

Revue des linguistes de l'université Paris X Nanterre

9 | 1997

Émile Benveniste. Vingt ans après

\section{Lectures de Benveniste : quelques variantes sur un itinéraire balisé}

\section{Claudine Normand}

\section{(2) OpenEdition}

Journals

Édition électronique

URL : http://journals.openedition.org/linx/964

DOI : $10.4000 /$ linx. 964

ISSN : 2118-9692

Éditeur

Presses universitaires de Paris Nanterre

Édition imprimée

Date de publication : 1 avril 1997

Pagination : 25-37

ISSN : 0246-8743

\section{Référence électronique}

Claudine Normand, «Lectures de Benveniste : quelques variantes sur un itinéraire balisé », Linx [En ligne], 9 | 1997, mis en ligne le 03 juillet 2012, consulté le 19 avril 2019. URL : http:// journals.openedition.org/linx/964

Ce document a été généré automatiquement le 19 avril 2019.

Département de Sciences du langage, Université Paris Ouest 


\title{
Lectures de Benveniste : quelques variantes sur un itinéraire balisé
}

\author{
Claudine Normand
}

1 C'est là un titre de randonneur qui écarte la flânerie comme l'aventure pour proposer une marche par étapes, autrement dit une entreprise sérieuse, supposant lecture de cartes et repérages de balises, ce qui n'exclut ni le plaisir de la découverte ni la possibilité de variantes.

2 Cet itinéraire annoncé pourrait être d'abord celui de Benveniste. On sait qu'il en affirme lui-même l'unité : unité de méthode et d'objectifs, situés dans une tradition, celle de grammairiens nourris de philologie avant tout indo-européenne, qu'ils travaillent à développer en linguistique générale. Meillet parlait avec la satisfaction d'un vieux maitre, de «l'Ecole de Paris » - expression de Robert Gauthiot - dont il soulignait la continuité de Bréal et Saussure à lui-même et ses élèves. C'était en 1928, à l'occasion d'un recueil de travaux offerts par ses amis au jeune Benveniste " pour son retour du service militaire ». Quarante ans plus tard, dans un entretien de 1968, Benveniste, interrogé sur sa longue production, la voit marquée par quelques thèmes personnels récurrents (« un certain nombre d'interrogations qui vous accompagnent pendant toute votre existence ») ${ }^{1}$ mais il ne revendique ni rupture par rapport à ses prédécesseurs ni originalité particulière parmi ses contemporains.

3 De fait, plus que Meillet, le modèle de Benveniste est Saussure, le linguiste indoeuropéaniste du Mémoire comme le linguiste philosophe du Cours. L'itinéraire de Benveniste peut être lu comme celui d'une fidélité à Saussure en même temps qu'un dépassement au sens hégélien du terme. Un objectif majeur de ses travaux, formulé à plusieurs reprises, est parfaitement résumé dans cet énoncé de 1966 :

Il nous incombe d'essayer d'aller au-delà du point où Saussure s'est arrêté dans

l'analyse de la langue comme système signifiant (II,219).

4 On pourrait aussi prendre un autre point de vue et s'attacher à l'itinéraire des lecteurs de Benveniste. Dans ce cas les variantes sont si importantes qu'on se demande parfois s'il s'agit du même parcours. Je ne parle pas seulement des interprétations mais du choix des textes. Ce qui se manifeste ici c'est la diversité de l'oeuvre qui suscite des lectures 
partielles parfois complètement disjointes; schématiquement on peut parler de trois types de lecture, à la fois en diachronie et en synchronie :

$51^{\circ}$ la lecture comparatiste, à la fois admirative et critique, privilégie les études indoeuropéennes de 1935, 1948 et 1969a et retient, évidemment, les travaux antérieurs (études iraniennes, études sur la langue ossète...) ; s'y ajoutent de très nombreux articles et comptes rendus produits tout au long de sa vie par Benveniste et dispersés dans des revues diverses de philologie et linguistique classiques; quelques-uns sont repris dans les Problèmes de Linguistique générale (PLG). C'est ici une lecture de philologues et de linguistes des langues classiques.

$62^{\circ}$ La lecture "structuraliste » : c'est plutôt la lecture des nouveaux - et alors jeunes linguistes des années 70 qui, se trouvant assez soudainement amenés à enseigner la linguistique à des étudiants, avaient grand besoin de s'informer eux-mêmes. On privilégie alors dans ce qui est facilement accessible, c'est à dire les deux volumes des PLG $(1966,1974)$, les articles où Benveniste présente des auteurs jugés «fondateurs » (Saussure, Bloomfield, Harris...), des notions «fondamentales» (signe, structure, niveaux...) et des synthèses sur les développements récents de la linguistique (cf. 1952c, 1954a, 1963a, 1968a,b...). Il s'agit de textes généraux, liés à la diffusion massive et tardive du structuralisme linguistique en France, en quelque sorte des articles de vulgarisation ou du moins visant une information initiale. J'y inclus cependant deux textes d'analyse plus approfondie: "Nature du signe linguistique» (1939) et "Niveaux de l'analyse linguistique » (1964).

$73^{\circ}$ Enfin la lecture de ce qui a été appelé «la théorie de l'énonciation »; suivant un regroupement adopté par Benveniste dans le premier volume des PLG, par ses éditeurs dans le second, elle se trouve associée aux thèmes «Communication » et «L'homme dans la langue ». Cette lecture ne débute réellement qu'à partir de 1970, mais devient alors, et durablement, dominante, sinon pour beaucoup exclusive ${ }^{2}$.

Cette dissociation de l'oeuvre qui se traduit souvent chez les différentes sortes de lecteurs par une ignorance ou une méfiance mutuelle, concerne sans doute peu un public de spécialistes, mais elle est sensible si on considère la plupart des lecteurs. Aucun linguiste français aujourd'hui n'ignore Benveniste, mais à quel Benveniste s'arrête-t-il? Je ne développerai pas davantage ici cette question qui serait importante à préciser dans une histoire de la réception de Benveniste en France (et de sa faible réception à l'étranger).

De fait ce que j'ai choisi de résumer ici c'est mon propre itinéraire dans ces textes; il reflète le parcours d'un linguiste ordinaire de ma génération et n'a pour particularité que d'avoir été longuement reparcouru jusqu'à ce colloque : parcours d'une linguiste de la fin des années 60 , pourvue par ses études d'une formation (légère) en grammaire comparée des langues classiques, formée de façon quasi autodidacte à la linguistique structurale dans les années 60 , et découvrant comme beaucoup la nouveauté de l'analyse de discours et des thèmes de l'énonciation dans les années 70. A chaque étape j'ai rencontré un Benveniste particulier, jusqu'à ce qu'il me paraisse souhaitable de mieux le connaître, plus précisément de chercher l'unité - s'il en est une - de cet homme apparemment divisé. Ce fut alors le début d'une assez longue marche, des premières années 80 jusqu'à aujourd'hui. Pour justifier cet angle de vue qu'on pourra trouver indûment personnel, j'invoquerai deux raisons : dans son cheminement hésitant ce rappel peut valoir comme témoignage, en quelque sorte historique, et comme tel, par les interprétations qu'il propose, il devrait ouvrir une discussion large et diverse. 
10 Dans le début des années 80 deux raisons m'ont poussée à m'intéresser à Benveniste : d'abord, en tant que disciple après-coup de Saussure, il intervenait dans l'approche globale qui était alors celle de notre groupe de recherche (GRHIL) ${ }^{3}$ : la réception et les interprétations de Saussure et du structuralisme en France; d'autre part, nous étions frappés par le retour en force, dans la linguistique française de ces années-là, du sujet, de la personne, de ce qui se formulait « la subjectivité dans le langage» dans des entreprises qui affirmaient toutes se fonder sur Benveniste, sa théorie du discours et de l'énonciation. Cette position à bien des égards paraissait contradictoire avec la fidélité proclamée de Benveniste à Saussure; elle était d'ailleurs présentée comme la grande nouveauté qui allait permettre de sortir enfin de la "clôture" structuraliste, de ce que C. KerbratOrecchionni allait bientôt appeler «l'étouffant confort de l'immanentisme $»^{4}$. Ainsi Benveniste qui s'affirmait sans cesse saussurien, devenait celui qui permettrait enfin de se délivrer du saussurisme; il devait nous débarrasser de ce qui nous avait paru le fondement même de la linguistique formelle, l'opposition langue / parole, la définition d'un ordre linguistique autonome, l'évacuation de la subjectivité. La perplexité du groupe se traduisit par un numéro de Langages $\left(n^{\circ} 77,1985\right)$, intitulé « Le sujet entre Langue et Parole(s) »; un bref avant-propos, sous l'intitulé « Le sujet est de retour » prenait acte, avec quelque distance, de cet état de fait et de la nécessité d'une réflexion.

11 Dans cet ensemble deux articles furent consacrés à la réception-interprétation de Benveniste. Dans le premier ( «L sujet dans la langue »), je présentais les résultats d'une enquête systématique portant sur les mentions, dans les articles de linguistique générale des années 60, des termes désignant l'énonciation et ses particularités, avec renvoi ou non à Benveniste. En effet, d'après mes souvenirs d'enseignante de ces années-là, les revues de linguistique n'attiraient pas spécialement l'attention sur ces textes devenus ensuite, semblait-il, emblématiques de Benveniste. S'agissait-il d'une insuffisance de lecture de ma part ou plus généralement d'une forme de résistance à la nouveauté de la part des linguistes? C'était ma question, immédiatement compliquée par l'observation suivante : trois de ces textes nouvellement pris en compte dataient respectivement de 1946, 1956 et 19585. Cet intérêt pour les marques de la personne et l'énonciation, qui paraissait la grande nouveauté des année 70, Benveniste, semblait-il, le manifestait bien avant; pourquoi n'avions-nous rien remarqué?

12 J'ai donc repris les numéros de Langages, qui était avec Langue française, la revue française la plus lue par les linguistes enseignants de cette période, plus la plupart des textes généraux d' "introduction ", « initiation », "présentation » de la linguistique générale, parus en nombre dans la deuxième moitié des années 60 . Résultat surprenant : personne, semblait-il, ne parlait de cet aspect de la pensée de Benveniste avant 1970. Cette affirmation est sûrement trop catégorique et demanderait une enquête complémentaire dans toutes les revues de linguistique françaises des années $60^{6}$; il reste, je crois, raisonnable de poser que ces notions, pourtant déjà largement développées par Benveniste, n'étaient pas mises en avant dans les années 60 , et quand il y était fait quelque allusion c'était en référence à Jakobson ${ }^{7}$ qui pourtant dans son article de 1957 sur les shifters renvoie explicitement à Benveniste. On parlait certes de Benveniste avant 1970 à propos de la linguistique synchronique, mais seulement de celui qui présentait les notions saussuriennes et les méthodes du structuralisme. Ce n'est qu'à partir de 1970 et du numéro 17 de Langages, "L'énonciation », dans lequel Benveniste produit son dernier article fameux, "L'appareil formel de l'énonciation», que les emplois des termes par lesquels il décrit le phénomène de l'énonciation se multiplient, mêlés à ceux de Jakobson, 
et que commence à s'élaborer une sorte de vulgate qu'on désigne par l'expression "Théorie de l'énonciation".

Mais si les linguistes ne prennent ces notions en compte que tardivement et d'abord en essayant de les introduire dans le cadre très différent de l'analyse de discours proposée par Harris, sur ce point Benveniste intéresse bien avant 1970 les philosophes et les psychanalystes. La deuxième partie de mon enquête me mettait sur cette voie: j'y comparais deux séries de notes de cours, prises à l'Université de Nanterre en 66-67, respectivement chez le linguiste J. Dubois et le philosophe P. Ricoeur. Alors que le linguiste évoque seulement le Benveniste structuraliste et ne le mentionne même pas quand il présente le fonctionnement des pronoms de personne, le philosophe expose l'essentiel de ce qui a été proposé à cette date sur la deixis et l'énonciation; il voit en Benveniste la promesse et déjà le commencement d'une tout autre linguistique, compagne naturelle de la phénoménologie.

C'est cette double détermination linguistique et phénoménologique des indicateurs qui rend possible le passage de la langue à la parole. En m'appropriant les signes vides du langage, je les applique à moi-même et à des situations du monde (Conclusion des notes prise au cours de Ricoeur).

J'en tirai la conclusion - qui rencontrait le point de vue dominant alors dans notre groupe - que la communauté linguistique avait eu besoin d'être sollicitée par son " extérieur », en particulier la philosophie, pour accepter les nouveautés dont elle était elle-même porteuse.

15 Ces résultats soulevaient évidemment beaucoup de questions qui m'ont poussée à lire Benveniste de façon plus large et plus précise. Ce qui me semblait important à éclairer (dans notre perspective historique) c'était l'élaboration de ces notions chez Benveniste lui-même, de voir à partir de quel moment on pouvait en repérer la présence dans ses recherches. Je me mis donc à travailler sur l'ensemble des PLG avec l'hypothèse plus ou moins claire que, malgré la présentation thématique en rubriques distinctes, il devait y avoir une unité de théorie et de méthode. En reprenant ces textes dans l'ordre chronologique de leur parution sur plus de trente ans (de 1939 à 1972), je pensais, avec quelque naïveté, pouvoir saisir la mise en place du thème de l'énonciation dans l'étude de la deixis. Le problème, à mes yeux, était triple :

1. l'analyse de l'énonciation se présente-t-elle comme une innovation chez Benveniste luimême?

2. si oui, comment se met-elle en place, comment s'introduisent les termes nouveaux ?

3. comment cette nouveauté a-t-elle été reçue et développée par les linguistes et les autres chercheurs en sciences humaines?

Prendre en compte l'ensemble des PLG me paraissait alors faire justice à l'unité de l'oeuvre, ce qui montre nos ignorances ou nos partis pris (j'englobe dans ce nous, ceux des linguistes généralistes qui veulent bien s'y reconnaître). Je savais évidemment, par mes souvenirs d'étudiante, que Benveniste avait écrit beaucoup plus que ce qui était retenu dans les Problèmes; ce travail de grammairien et de philologue, à ce moment là, je n'ai simplement pas pensé à l'intégrer dans une recherche qui se voulait pourtant globale. Les observations et conclusions de cette étude restreinte (présentées à l'occasion d'un colloque sur l'histoire de l'énonciation en 1985) peuvent se résumer ainsi : dans mon enquête sur l'apparition soudaine ou progressive de la terminologie de l'énonciation, je n'avais $\mathrm{pu}$ déceler dans aucun texte de changement décisif; mon hypothèse discontinuiste ne marchait pas. Mais, ce que je n'attendais pas et qui alors me frappa dans 
cette lecture continue, c'est l'alternance de deux types de textes différenciés, relevant de deux démarches et de deux rhétoriques: d'une part des analyses de phénomènes linguistiques particuliers, morpho-syntaxiques ou lexicaux, auxquels était appliquée chaque fois la même méthode : une description minutieuse d'une structure particulière, dans une ou plusieurs langues, se concluant par une généralisation à visée explicative ; ainsi la description des relations de personne (1946), de la phrase nominale (1950b), de la voix moyenne par rapport à la voix active (1950a) de la phrase relative (1957), etc... D'autre part des textes entièrement théoriques se présentant, selon les cas, comme des bilans ou des programmes, d'un ton généralement dogmatique : ils énoncent une série d'affirmations de portée générale, dans une argumentation qui se veut serrée; ils traitent de la linguistique générale dont ils font le bilan ou dessinent les tendances «actuelles", puis, de plus en plus de la sémiologie, telle qu'elle existe et telle qu'elle doit advenir. Je regroupe dans cet ensemble les textes sur Saussure et le structuralisme (1954, 1963a, 1968a), «Langue et société »(1965), «Le langage et l'expérience humaine (1965b), «La forme et le sens dans le langage »(1966), et « La sémiologie de la langue » (1969); on peut y inclure l'article de 1939 sur la «Nature du signe linguistique» et celui de 1964, « Niveaux de l'analyse linguistique ». Dans tous ces textes sont avancées des propositions conceptuelles sur le langage et la langue, des distinctions devenues célèbres telles que langue/discours, sémiotique /sémantique et la double signifiance qui sont associées, d'une façon de plus en plus nette, à la présentation programmatique de la sémiologie, science générale des signes. C'est dans ce type de textes que Benveniste affirme reprendre les questions là où Saussure les a laissées, pour aller, non pas ailleurs mais plus loin :

Reprenant ce grand problème où Saussure l'a laissé nous voudrions insister d'abord sur la nécessité d'un effort préalable de classement si l'on veut promouvoir

l'analyse et affermir les bases de la sémiologie, (1969, II,50);

"Promouvoir l'analyse ", c'est ce qu'il faisait en praticien dans les analyses concrètes où il appliquait strictement les principes saussuriens ; " affermir les bases de la sémiologie » en tant que science générale, qu'il dit parfois "universelle", c'est ce qu'il cherchait à formuler dans ces programmes de plus en plus vastes où se dessinait une science générale de la culture.

Frappée par la dualité de ces textes je les opposai comme deux "postulations» de Benveniste: d'un côté un linguiste aux prises avec la description toujours reprise, inachevable, des structures formelles, des configurations concrètes de telle(s) langue(s) particulière(s); de l'autre un théoricien du langage, désireux de synthétiser dans une totalité d'inspiration toute philosophique les traits constitutifs de la nature du langage, de la communication, du discours, de la langue, de tout cela à la fois qu'on appelle aujourd'hui volontiers le langagier. Je résumai cette dualité dans une formulation métaphorique en opposant textes $\mathrm{d}^{\prime}$ ' ouverture » et textes de "fermeture » : ouverture des analyses concrètes, suscitant d'autres analyses à venir, parce que la description est inachevable ; c'est bien ce que dit l'article de 1970, «L'appareil formel de l'énonciation », que j'hésite à classer dans les textes-bilans à cause de sa conclusion: De longues perspectives s'ouvrent à nous, à partir du cadre formel esquissé ici (II, 88); fermeture des bilans et des programmes, achevant la théorie dans une totalisation de forme philosophique: ainsi le texte de 1969, promettant une sémiologie «de deuxième génération » devant concourir au développement de la "sémiologie générale (1969b, II, 66). L'unité de Benveniste m'apparaissait une unité divisée, celle d'un philosophe (d'inspiration hégélienne), contrarié par le linguiste qui ne peut épuiser la richesse et la complexité de 
son objet. Même si les traits en sont un peu forcés, je continue à trouver cette opposition éclairante et je la propose comme telle à la discussion.

Quant à ma question de départ (comment la théorie de l'énonciation a-t-elle été mise en place par Benveniste? par quelles étapes ?) elle devenait : les éléments théoriques de la deixis, ce rôle de la subjectivité dans le langage, ne sont-ils pas présents dès le début de ses travaux, opérateurs de toute sa démarche de linguiste? Leur systématisation ne seraitelle pas le fait d'abord de non-linguistes (philosophes, psychanalystes, théoriciens de la littérature...) ce qui expliquerait que Benveniste lui-même ne se soit préoccupé que tardivement d'en proposer une théorie d'ensemble?

A l'appui de cette suggestion on peut faire remarquer que l'expression «sujet d'énonciation" n'a jamais été employée par Benveniste lui-même; ce sont ses commentateurs philosophes et psychanalystes, semble-t-il, qui ont fabriqué et répandu très tôt ce terme (en particulier J. Kristeva et J. Lacan). Ce détail n'est pas seulement anecdotique; en fait le mot sujet désigne dans ces textes, selon les cas ou indistinctement, le sujet grammatical, le sujet psychologique ou encore l'ego philosophique, revu par la phénoménologie et repris souvent sous la figure de la personne, mais jamais une entité qui pourrait faire penser au sujet « clivé » de la psychanalyse ; si bien que, lorsque Benveniste décrit les traces linguistiques de la personne et de l'intersubjectivité, il enrichit la description linguistique mais n'apporte rien, me semble-t-il, d'immédiatement intégrable à une théorie du sujet d'inspiration psychanalytique, telle qu 'elle se cherchait dans ces années-là.

21 Ce que je remarquais cependant (que j'ai développé surtout ensuite) c'est que se nouaient, au coeur de ses descriptions linguistiques, les notions de sujet (avec tout le flou mentionné) et de signification. Cette liaison se retrouve dans tous les textes; elle n'est pas réservée à ceux qui analysent explicitement les termes de la deixis. C'est par elle qu'est résumée et sémantiquement fondée la configuration formelle qui fait l'objet de telle ou telle description, selon un principe constamment affirmé :

...encore faut-il commencer par voir au-delà de la forme matérielle et ne pas faire tenir toute la linguistique dans la description des formes linguistiques. (1952b,I,118)

Le plus souvent ce fondement est formulé métaphoriquement dans l'opposition d'un intérieur, domaine du sujet, et d'un extérieur, le monde objectif. La diversité des formes linguistiques signifie (c'est à dire à la fois constitue et formule) la diversité des relations entre le sujet et le monde. Les exemples sont très nombreux, je n'en citerai que deux: opposant l'actif et le moyen Benveniste affirme :

(La diathèse) dénote une certaine attitude du sujet relativement au procès par où ce procès se trouve déterminé dans son principe;(...) Dans l'actif les verbes dénotent un procès qui s'accomplit à partir du sujet et hors de lui ; dans le moyen (...) le verbe indique un procès dont le sujet est le siège; le sujet est intérieur au procès. (1950a,I,170.172)

et sur la structure du verbe auxiliaire :

On arrive ainsi à définir la situation respective de être et avoird'après la nature du rapport institué entre les termes nominaux de la construction: être présente une relation intrinsèque, avoir une relation extrinsèque $(1965, \mathrm{II}, 187)$

Il s'ensuit que les textes « canoniques » concernant l'énonciation ne semblent pas avoir de statut particulier dans l'oeuvre de Benveniste. Comme les autres textes ils font intervenir dans la description des phénomènes linguistiques les paramètres du sujet et de la signification et on peut y repérer le même clivage entre analyses concrètes et textes théoriques ; les textes de 1946, 56, 59 sur la personne et le temps et ceux de 1958, 66, 69 sur 
la subjectivité, la sémiologie, la forme et le sens présentent ce type de différence qu'on trouve également entre les deux textes de 1965, «L'antonyme et le pronom en français » et «Le langage et l'expérience humaine ». La particularité des textes dits de l'énonciation n'est donc pas de prendre en compte la subjectivité mais de l'étudier dans ses indices les plus directement visibles et, à partir de là, d'en faire une étude linguistique systématique servant de fondement privilégié à la théorie de ce que Benveniste appelle tantôt le sémantique, tantôt la sémiologie .

J'ai bien conscience que de telles affirmations que je ne peux qu'énoncer ici sans les argumenter par le détail des textes, appellent discussion et rectification éventuelle. C'est en tant que telles que je les ai trouvées propres à un exposé introductif. Je le terminerai en indiquant simplement les deux directions que j'ai suivies par la suite et en formulant quelques unes des questions que j'ai rencontrées.

Une direction a été de chercher à préciser ce que Benveniste entend par sémiologie dans les textes où il en présente le programme ou un bilan. On y retrouve l'ambiguïté de l'opposition extérieur / intérieur par laquelle Saussure définissait le rapport de la linguistique aux autres sciences d'une part, à la sémiologie de l'autre. On sait que pour Saussure la linguistique prenait place dans le cercle des différentes sciences du signe (l'image du cercle, absente du CLG est insistante dans les manuscrits), cercle qui renvoie les autres sciences dans un extérieur; en même temps, à l'intérieur même du cercle sémiologique, une autre partition détache radicalement une linguistique interne et une linguistique externe; par là est avancé le principe de ce que l'on a appelé ensuite l'immanentisme. Benveniste, parti de cette position immanente pose, dès 1954, la nécessité de son élargissement en disant du CLG qu'il « projette la langue sur le plan d'une sémiologie universelle » (I, 7); dans trois articles de 1963 il reprend ce projet qu'il appelle tantôt " véritable science de la culture ", tantôt " sémiologie générale »; le mouvement s'amplifie jusqu'à la "sémiologie de 'deuxième génération' » du texte de 1969, qui doit nous permettre de "dépasser la notion saussurienne du signe comme principe unique " et contribuer au « développement des autres branches de la sémiologie générale » (II, 66).

Dans ce mouvement d'intégration des sciences sous l'égide de la linguistique, l'extérieur semble progressivement résorbé par un intérieur devenu hypertrophique. Le monde entier devient tissu de signes, texte porteur de sens ${ }^{9}$. On peut comprendre et même partager ce fantasme tout en posant quelques questions à la cohérence des textes; par exemple: quelles relations précises entretiennent d'une part les termes sémiologie et sémiotique, d'autre part sémiologie et sémantique? En effet dans le texte de 1969 c'est à partir de ce que Benveniste appelle désormais le sémantique ou linguistique du discours que se déploie cette sémiologie universelle. Une autre question à ce sujet pourrait porter sur le rapport de ce programme à celui de la sémiotique positiviste américaine, Peirce sans doute mais surtout Morris et Carnap; je rappelle que cette sémiotique se présente explicitement chez Morris comme la réalisation d'une logique renouvelée intégrant la pragmatique, «nouvel organon», en quelque sorte une science des sciences. Mais on peut aussi bien interroger ce programme sur son rapport à la phénoménologie, philosophie du sens et du sujet ${ }^{10}$. On dira que les deux références semblent s'exclure, mais Benveniste mentionnant à peine Peirce et Morris et jamais, à ma connaissance, son collègue au Collège de France, M. Merleau-Ponty, ne nous éclaire pas sur ses préférences philosophiques.

Dans une direction assez différente je me suis attachée au rapport de Benveniste à Saussure dans sa méthode descriptive, prenant cette fois en compte, outre les textes des 
PLG, une partie des textes rattachés plus haut à la « lecture comparatiste », en particulier les études indo-européennes de 1935 et $1948^{11}$. Benveniste m'est apparu alors comme le plus saussurien des linguistes dans la mesure où, ne séparant jamais signifiant et signifié, il affirme sans cesse la nécessité, dans l'étude des structures, de rattacher l'analyse de la configuration formelle à une fonction sémantique, ce qu'il fait dans chaque cas tout en renvoyant cette fonction sémantique au rôle d'un sujet, et cela dès Noms d'agent, noms d'action:

L'existence de deux types de noms d'agent (...) peut se réaliser (...) chaque fois qu'on veut opposer dans la désignation du sujet agissant, des modes d'action sentis comme distincts (66).

Cette interprétation par les particularités du sujet semble assez loin de Saussure; on pourrait également s'interroger sur sa conception de l'arbitraire du signe. Il semble que sur ce point Benveniste soit fidèle sans doute au comparatisme (où l'arbitraire du signe est un postulat) mais qu'il n'adopte pas vraiment toutes les implications de ce principe chez Saussure. Or l'interprétation faite de l'arbitraire engage une position en sémantique et c'est bien la sémantique que vise Benveniste ${ }^{12}$. Je réserve ce point à la discussion et je conclurai de façon abrupte en caractérisant l'unité de Benveniste comme celle d'une obsession : qu'il s'agisse de surmonter les oppositions réductrices qui sont au départ du champ linguistique et sémiologique moderne ou de trouver une explication à des anomalies formelles, ce qu'il manifeste est toujours le souci de retrouver une unité. Dans les descriptions linguistiques cette méthode produit des éclairages nouveaux qui sont parfois éblouissants mais laissent, paraît-il, dans l'ombre certaines difficultés ; les textes théoriques quant à eux ne laissent pas de rappeler l'ambitieux projet épistémologique de la fin du XIX siècle auquel est attachée le nom de Dilthey : fonder, face au positivisme, une épistémologie propre des sciences humaines; la démarche philosophique veut s'assurer ici du sérieux scientifique que donne la spécialisation. On comprend que Ricoeur en ait été séduit. Quant à nous, comme disait R. Barthes " nous lisons d'autres linguistes (il le faut bien) mais nous aimons Benveniste $»^{13}$.

\section{BIBLIOGRAPHIE}

Emile BENVENISTE : Ouvrages et présentation chronologique des articles repris dans les Problèmes de linguistique générale.

BenVeniste, E. (1935) Origine de la formation des noms en Indo-Européen, Maisonneuve, Paris

BenVeniste, E. (1948) Noms d'agent et noms d'action en Indo-Européen, Maisonneuve, Paris

BenVeniste, E. (1966) Problèmes de linguistique générale, Gallimard, Paris

BenVEnISTE, E. (1969) Le vocabulaire des institutions indo-européennes, Minuit,Paris

BenVeniste, E. (1974) Problèmes de linguistique générale, II, Gallimard, Paris

BenVeniste, E. (1939) « Nature du signe linguistique », Acta linguistica ; 1966, ch. IV 
BENVENISTE, E. (1946) « Structure des relations de personne dans le verbe », BSL, XLIII, fasc. 1 ; $1966, \mathrm{ch}$. XVIII

BenVeniste, E. (1949a) «Le système sublogique des prépositions en latin », Travaux du Cercle linguistique de Prague, vol. V, Recherches strusturales ; 1966, ch.XIII

BenVENISTE, E. (1949b) « Euphémismes anciens et modernes », Die Sprache,I, 949 (=Festschrift W. Havers) ; 1966, ch.XXV

BenVeniste, E. (1950a) « Actif et moyen dans le verbe », Journal de Psychologie,43,1950 ; 1966, ch. XIV

BENVENISTE, E. (1950b) « La phrase nominale », BSL XLVI, fasc.I ; 1966, ch. XIII

BENVENISTE, E. (1951a) « Don et échange dans le vocabulaire indo-européen », Année sociologique, $3^{\circ}$ série, t.II, PUF $1951 ; 1966$, ch.XXVI

BENVENISTE, E. (1951b) « La notion du rythme dans son expression linguistique », Journal de psychologie, 44, 1950 ; 1966, ch. XXVII

Benveniste, E. (1952a) « Communication animale et langage humain », Diogène I, Nov.1952 ; 1966, ch. V

BenVENISTE, E. (1952b) « La classification des langues » Conférences de l'Institut de Linguistique de l'Université de Paris ; 1966, ch. IX

BENVENISTE, E. (1952c) « La construction passive du parfait transitif », BSL, 48,1952, fasc. 1 ; 1966, ch. XV

BENVENISTE, E. (1954a) « Tendances récentes en linguistique générale », Journal de psychologie, $1954 ; 1966$, ch. I

BenVENISTE, E. (1954b) «Problèmes sémantiques de la reconstruction », Word, vol. X, n ${ }^{\text {os }}$ 2-3, $1954 ; 1966$, ch. XXIV

BENVENISTE, E.(1954c) «Civilisation : contribution à l'histoire du mot », Hommage à Lucien Febvre, Paris $1954 ; 1966$, ch. XXVIII

BENVENISTE, E. (1956a) « Remarques sur la fonction du langage dans la découverte freudienne », La Psychanalyse I, $1956 ; 1966, \mathrm{ch}$. VII

BenVeniste, E. (1956b) « La nature des pronoms », For Roman Jakobson, La Haye : Mouton \& $C^{\circ}$, $1956 ; 1966$, ch.XX

BENVENISTE, E. (1957) «La phrase relative, problème de syntaxe générale », BSL, LIII, 1957-58, fasc. $1 ; 1966$, ch.XVII

BenVeniste, E. (1958a) «Catégories de pensée et catégories de langue », Les Etudes philosophiques, 4 oct.-déc. $1958 ; 1966$, ch.VI

BenVeniste, E. (1958b) « De la subjectivité dans le langage », Journal de psychologie, 55, 1958 ; 1966, ch.XXI

BenVENISTE, E. (1958c) «Les verbes délocutifs », Studia philologia et litteraria in Honorem L. Spitzer, Bern : Francke, $1958 ; 1966$, ch.XXIII

BENVENISTE, E.(1959) «Les relations de temps dans le verbe français », BSL, 54, 1959, fasc.1; 1966, ch.XIX 
BENVENISTE, E.(1960) «"être et "avoir" dans leur fonction linguistique », BSL, 54, 1960, fasc. $1 ; 1966$ ch.XVI

BENVENISTE, E. (1962a) «"Structure" en linguistique », Sens et usage du terme structure dans les sciences humains et sociales (R. Bastide ed.), La Haye, Mouton, $1962 ; 1966$ ch. VIII.

BenVeniste, E. (1962b) « Pour l'analyse des fonctions casuelles : le génitif latin », Lingua, vol. XI, Amsterdam ; 1966, ch.XII

Benveniste, E. (1963a). « Coup d'oeil sur le développement de la linguistique », Académie des Inscriptions et Belles-Lettres, Nov. 1962, paru en 1963 ; 1966 I, ch. II

BENVEniste, E. (1963b) « Saussure après un demi-siècle », Cahiers Ferdinand de Saussure, 20, 1963 ; 1966, ch.III

BenVEniste, E. (1963c) « Diffusion d'un terme de culture : latin orarium », Studia classica et orientalia Antonio Pagliaro oblata, Instituto di Glottologia di Roma, vol.I ; 1974, ch.XVI

BenVEniste, E. (1963d) «La philosophie analytique et le langage », les Etudes philosophiques, 1963, n ${ }^{\circ} 1 ; 1974$, ch.XXII

BenVeniste, E. (1964) « Les niveaux de l'analyse linguistique », Proceedings of the ninth International Congress of Linguists (1962), Camb.Mass., La Haye, Mouton \& $C^{\circ} ; 1974$, ch. X

BenVeniste, E. (1965a) « Structure des relations d'auxiliarité », Acta Linguistica Hafnensia, 9, 1965 ; 1974, ch.XIII

BenVeniste, E. (1965b) « Le langage et l'expérience humaine », Diogène, 51 (juil.-sept. 1965) ; 1974, ch. XIV

BENVENISTE, E. (1965c) «L'antonyme et le pronom en français moderne », BSL, 60, 1965, fasc. 1 ; 1974, ch.XIV

BenVeniste, E. (1966a) « La forme et le sens dans le langage », Le Langage, 11, Neuchâtel : La Baconnière, 1967 (Société de Philosophie de langue française, Actes du XIII ${ }^{\circ}$ congrès, Genève, 1966) ; 1974, ch.XV

BenVeniste, E. (1966b) «Convergences typologiques », l'Homme, 6, 1966, Cahier 2;1974, ch.VII

BeNVENISTE, E. (1966c) «Formes nouvelles de la composition nominale », BSL, 61, 1966, fasc. 1 ; 1974 , ch.XII

BENVENISTE, E.(1966d) « Les transformations des catégories linguistiques » (publié en traduction anglaise dans Directions for historical linguistics, Symposium in Historical Linguistics, April 1966, University of Texas Press, $1968 ; 1974$, ch.IX

BenVEniste, E. (1966e) Problèmes de linguistique générale, Paris, Gallimard

BenVeniste, E. (1968a) « Structuralisme et Linguistique », un entretien de P. Daix avec E. Benveniste, Les Lettres françaises, 1242, juillet $1968 ; 1974$, ch. I

BenVeniste, E. (1968b) «Ce langage qui fait l'histoire », propos recueillis par G. Dumur, le Nouvel Observateur, 20 nov.au 20 déc.1968; 1974, ch.II

BENVENISTE, E. (1968c) «Structure de la langue et structure de la société », Linguaggi nella società $i$ nella tecnica, Milan, Edizioni di Communità, 1970, Convegno internazionale Olivetti, 1968 ; 1974, ch.VI

BENVENISTE, E. (1969a) Le vocabulaire des institutions indo-européennes, Minuit,Paris 
BenVeniste, E. (1969b) « Sémiologie de la langue », Semiotica, I ; 1974, ch III

BenVeniste, E. (1969c) « Mécanismes de transposition », Cahiers Ferdinand de Saussure, 23, 1969 (= Mélanges H.Frei) ; 1974, ch.VIII

BenVeniste, E. (1969d) « Comment s 'est formée une différenciation lexicale en français », Cahiers Ferdinand de Saussure, 22, 1966 ;1974, ch.XIX

BenVeniste, E. (1969e) « Genèse du terme "scientifique" ", l’Age de la Science, I, Aix 1969;1974, ch. XV.

BenVeniste, E. (1969f) « La blasphémie et l'euphémie », Archivio di Filosofia, Rome 1969; 1974, ch. XVIII.

BENVENISTE, E. (1970a) « L'appareil formel de l'énonciation », Langages, 17, Mars $1970 ; 1974$ ch.V

BenVeniste, E. (1970b) « Deux modèles linguistiques de la cité », Mélanges offerts à Claude LéviStrauss à l'occasion de son soixantième anniversaire, La Haye, Mouton, 1970 ; 1974, ch. XX

BenVeniste, E. (1972) « Pour une sémantique de la préposition allemande Vor » Athenaeum, nouvelle série, vol. I, fasc. III-IV, Université de Pavie, 1972 ; 1974, ch.X

\section{Ouvrages cités}

BARTHES, R. (1974) Le bruissement de la langue, Seuil, Paris

COQUET, J.C. (1992) « Notes sur Benveniste et la phénoménologie », LINX, 26, p.41-48

JAKoBson, R. (1957) « Shifters, Verbal Categories, the Russian Verb », Russian Language Project, Harvard University ; tr. dans Essais de Linguistique Générale, ch. IX, 1963, Minuit, Paris

Kerbrat-Orecchioni, C. (1986) L'implicite, Colin, Paris

MeILlET, A. (1928) Etrennes linguistiques offertes par quelques amis à Emile Benveniste, Champion, Paris NoRMAND, Cl. (1985a) « Le sujet dans la langue »,Langages,77, p7-21

NORMAND, Cl. (1985b) «Linguistique et philosophie : un instantané dans l'histoire de leurs relations ", Langages 77, p.33-43

Normand, Cl. (1986) «Les termes de l'énonciation chez Benveniste », Histoire, Epistémologie, Langage, t8, fasc. II, p. 191.206

Normand, Cl. (1989) «Constitution de la sémiologie chez Benveniste », Histoire, Epistémologie, Langage t 11, fasc. II, p. 141-169

NoRMAND, Cl. (1992) «Benveniste : linguistique saussurienne et signification », LINX, 26, p.49-75

NoRmand, Cl. (1996) «E.Benveniste : quelle sémantique ?», LINX, Du dire et du discours. Hommage à Denise Maldidier, p. 221-238

\section{NOTES}

1. 1968 b/II,37.Dans la suite les références sont données dans le cours du texte et dans cet ordre : date de parution, volume des PLG,page dans le volume.

2. Cf. Normand 1985. 
3. Le GRHIL (Groupe de recherche en histoire de la linguistique),rattaché depuis 1984 à l'URA CNRS 381, est une structure amicale, constituée en 1976 en vue du numéro 49 de Langages "Saussure et la linguistique pré-saussurienne »; il a été depuis le point de départ de nombreuses publications.

4. 1986, p. 149.

5. Cf. Biblio.1956b et 1958 b.

6. J'ai trouvé en particulier par la suite une mention des articles de 1946, 56 et 58 chez Peytard, citant Genette (Communications 12,1968) dans le numéro de La Nouvelle Critique consacré à « Linguistique et Littérature », 1968.

7. Ainsi chez Dubois et Sumpf, dans le même numéro de Communications.

8. Cf. Normand 1986.

9. Cf. Normand 1989.

10. Cf. J.C. Coquet 1992.

11. Cf. Normand 1992.

12. Cf. Normand 1996

13. R. Barthes 1974.

\section{RÉSUMÉS}

Partant d'une particularité de la réception de l'oeuvre de Benveniste en France - des lectures disjointes, ne s'attachant dans chaque cas qu'à une partie d'une oeuvre très diverse - l'auteur expose son propre itinéraire dans ces textes, sur plus de dix ans, Cette histoire d'une lecture progressivement élargie, rectifiée, approfondie et toujours ouverte, propose une interprétation de l'unité problématique de l'oeuvre de Benveniste.

Starting from the fact that the interest of readers is generally limited to a part of Benveniste's work, the author describes her own path, for more than ten years, within these texts. This is the story of an interpretation ever corrected and broadened, of a difficult to grasp unity of thought. 